Manuscript received 08.05.2020

Accepted: 30.06.2020

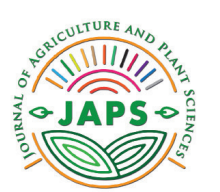

\author{
In print: ISSN 2545-4447 \\ On line: ISSN 2545-4455 \\ doi: https://doi.org/10.46763/JAPS2010017g \\ Professional paper
}

\title{
EFECTS OF HERBICIDES ON BARLEY SEEDS GERMINATION DEPENDING ON GROWTH STAGE
}

\author{
Gordana Glatkova ${ }^{1 *}$, Mentor Zekiri² \\ 'University "Ss Cyril and Methodius", Institute of Agriculture-Skopje, Republic North Macedonia \\ ${ }^{2}$ Ministry of Agriculture, Forestry and Water Economy, Republic North Macedonia \\ *Corresponding author: gordana glatkova@yahoo.com
}

\begin{abstract}
An experiment was carried out including three barley varieties (Reks, NS 293 and Egej), treated with six herbicides (2,4-D, MCPP+dicamba, triasulfuron+dicamba, 2,4-D+florasulam, amidosulfuron+iodosulfuron and florasulam+flumetsulam) in the three different growth stages (tillering, first node and second node). The aim of this experiment was to consider the influence of herbicides (applied in different growth stages) on germination of barley seeds.

In all experimental years herbicides did not significantly influence barley seed germination. The growth stage during herbicide application did not affect germination of barley. If conditions do not allow herbicides to be used in the optimal period, it can be used until second node stage, without having a negative impact on the barley seeds germinations.
\end{abstract}

Key words: barley, varieties, herbicides, growth stages, seeds germination

\section{INTRODUCTION}

Barley (Hordeum sativum Jessen) in the Republic North Macedonia is grown on about 47500 ha with average yield of $3440 \mathrm{~kg} / \mathrm{ha}$ Anonymous (2009). It is the second cultivated crop right after the wheat. It is grown as continuous crop two to three years. Because of that, barley production is disturbed by weed infestation. In barley losses due to competitive effects of weeds estimated at $15-25 \%$ of potential production. Contemporary chemical weed control in barley and wheat begins after Second World War. Since then over 50 a. i. are synthesized for selective weed control in barley and wheat. Most of the herbicides which are used in barley and wheat is foliar and tillering is optimum growth stage for application. According Folley (1985), barley is more sensitive to herbicides than wheat. Various herbicides has various influence on barley, dependent on barley varieties and the growing stages during the application. Tottman (1976), emphasizes that the knowing of growth stages during the herbicides application is of the high importance. By using the herbicides in advance growth stages barley sensitivity can be increased and barley yield elements can be reduced Allien (1966), Markovich (1978), (Rinella et al, 2001). (Friesen et al.,1964, 1968) found that in cereals the negative influence of dicamba was increased if it is applicated in later growth stages. Barley seed production in North Macedonia is present on 1019 ha with average yield of $6000 \mathrm{~kg} / \mathrm{ha}$. Seed germination of barley is very important for quality seed production and also, for determining correct seeding rate.

There is little data about the impact of herbicides on wheat and barley seed germination. Randy (1986), emphases that wheat seed germination was not affected by metribuzine and chlorsulfuron and their combination, but metribuzine when applied alone reduced coleoptile growth of Vona variety seed.

According to this an experiment was carried out to consider the influence of herbicides on barley seeds germination depend on growth stage. 


\section{MATERIAL AND METHODS}

Field trial was conducted at the Agriculture institute in Skopje. The experimental design was randomized complete block with four replicates, and harvest plot size of $16 \mathrm{~m}^{2}$. The trial was three factorial (Factor 1-herbicides, Factor 2-varieties and Factor 3-barley growth stages during herbicides application). The studies were carried

out with three barley varieties Reks, NS 293 and Egej which were seeded with seedling rate of $300 \mathrm{~kg} / \mathrm{ha}$ on October $19^{\text {th }}\left(1^{\text {st }}\right.$ year $)$, November $4^{\text {th }}\left(2^{\text {nd }}\right.$ year) and November $13^{\text {th }}$ ( 3 rd year). The harvest was carried out with plot combine Wintersteiger on June $22^{\text {th }}\left(1^{\text {st }} y e a r\right)$, July $3^{\text {th }}\left(2^{\text {nd }}\right.$ year) and July $18^{\text {th }}$ ( $3^{\text {rd }}$ year).

Table 1. Variants of the trial.

\begin{tabular}{|l|c|c|}
\hline Variants - active ingredient (a.i.) & Rate & Time of application \\
\hline Weed free control & $/$ & $/$ \\
\hline $2,4-D$ & $1 \mathrm{~L} / \mathrm{ha}$ & $\mathrm{I}, \mathrm{II}, \mathrm{III}$ \\
\hline MCPP+dicamba & $4 \mathrm{~L} / \mathrm{ha}$ & $\mathrm{I}, \mathrm{II}, \mathrm{II}$ \\
\hline Triasulfuron+dicamba & $100 \mathrm{~g} / \mathrm{ha}$ & $\mathrm{I}, \mathrm{II}, \mathrm{II}{ }^{*}$ \\
\hline 2,4-D+florasulam & $0.5 \mathrm{~L} / \mathrm{ha}$ & $\mathrm{I}, \mathrm{II}, \mathrm{II}$ \\
\hline Amidosulfuron+iodosulfuron & $0.25 \mathrm{~kg} / \mathrm{ha}$ & $\mathrm{I}, \mathrm{II}, \mathrm{III}$ \\
\hline Florasulam+flumetsulam & $60 \mathrm{~m} \mathrm{~L} / \mathrm{ha}$ & $\mathrm{I}, \mathrm{II}, \mathrm{II}$ \\
\hline
\end{tabular}

*I-tillering, II- first node, III- second node

All herbicides were applied with $\mathrm{CO}^{2}$-pressurized backpack sprayer with $300 \mathrm{~L} / \mathrm{ha}$ water.

Seeds germination was measured according to ISTA methods. The data were subjected to statistical analysis applying LSD-test.

\section{RESULTS AND DISCUSSION}

In $1^{\text {st }}$ year (Table 2) barley seed germination was ranged from $96,0 \%$ at NS 293 variety treated with 2,4D+florasulam at second node stage to $97,8 \%$ at Reks variety treated

with MCPP+dicamba at tillering stage. The investigated herbicides did not significantly influence the barley seed germination.

Table 2. Influence of herbicides on barley seeds germination ( $1^{\text {st }}$ year).

\begin{tabular}{|c|c|c|c|c|c|c|c|c|c|}
\hline \multirow[t]{4}{*}{ Variants } & \multicolumn{9}{|c|}{ Varieties } \\
\hline & \multicolumn{3}{|c|}{ Reks } & \multicolumn{3}{|c|}{ NS 293} & \multicolumn{3}{|c|}{ Egej } \\
\hline & 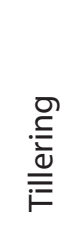 & 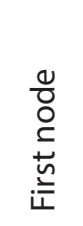 & 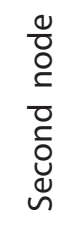 & 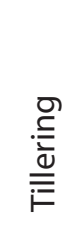 & 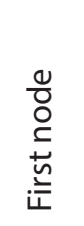 & 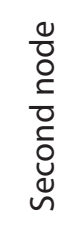 & 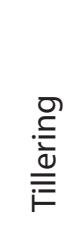 & 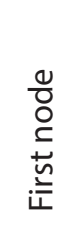 & 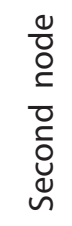 \\
\hline & $\%$ & $\%$ & $\%$ & $\%$ & $\%$ & $\%$ & $\%$ & $\%$ & $\%$ \\
\hline Weed free control & 97.3 & 97.3 & 97.3 & 96.0 & 96.0 & 96.0 & 96.8 & 96.8 & 96.8 \\
\hline $2,4-D$ & 96.3 & 96.3 & 96.5 & 96.5 & 96.8 & 96.8 & 96.8 & 97.5 & 96.5 \\
\hline MCPP+dicamba & 97.8 & 96.3 & 96.5 & 97.3 & 97.0 & 97.3 & 96.5 & 97.0 & 96.0 \\
\hline Triasulfuron+dicamba & 96.5 & 97.0 & 96.3 & 96.8 & 96.8 & 96.5 & 96.8 & 96.3 & 96.5 \\
\hline 2,4-D+florasulam & 96.5 & 96.3 & 96.5 & 97.0 & 96.5 & 96.0 & 96.5 & 96.3 & 97.5 \\
\hline Amidosulfuron+iodosulfuronn & 96.3 & 96.3 & 97.0 & 96.3 & 97.3 & 97.0 & 97.0 & 97.3 & 96.8 \\
\hline Florasulam+flumetsulam & 96.5 & 96.5 & 97.0 & 96.8 & 97.3 & 97.0 & 96.8 & 96.3 & 97.3 \\
\hline LSD 0.05 & 1.7 & 2.2 & 2.0 & 1.4 & 1.3 & 1.6 & 1.9 & 1.9 & 1.9 \\
\hline LSD 0.01 & 2.3 & 3.0 & 2.7 & 2.0 & 1.8 & 2.1 & 2.5 & 2.5 & 2.6 \\
\hline
\end{tabular}


In $2^{\text {nd }}$ year (Table 3 ) the highest barley seed germination $(98,8 \%)$ was measured at NS 293 variety treated with amidosulfuron + iodosulfuron at tillering stage. Lowest barley seed germination $(97,0 \%)$ was measured at NS 293 variety treated with 2,4-D at first and second node stage and amidosulfuron+iodosulfuron at first node stage, also at Egej variety treated with 2,4-D+florasulam and florasulam+flumetsulam at first node stage. The investigated herbicides did not significantly influence the barley seed germination.

Table 3. Influence of herbicides on barley seeds germination ( $2^{\text {nd }}$ year).

\begin{tabular}{|c|c|c|c|c|c|c|c|c|c|}
\hline \multirow{4}{*}{ Variants } & \multicolumn{9}{|c|}{ Varieties } \\
\hline & \multicolumn{3}{|c|}{ Reks } & \multicolumn{3}{|c|}{ NS 293} & \multicolumn{3}{|c|}{ Egej } \\
\hline & 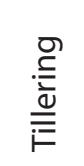 & 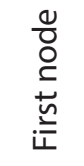 & 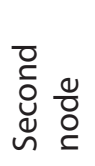 & 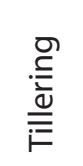 & 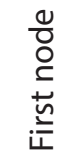 & 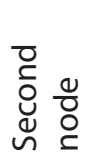 & 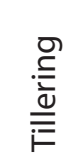 & 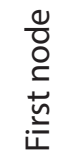 & 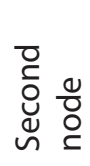 \\
\hline & $\%$ & $\%$ & $\%$ & $\%$ & $\%$ & $\%$ & $\%$ & $\%$ & $\%$ \\
\hline Weed free control & 97.8 & 97.8 & 97.8 & 98.0 & 98.0 & 98.0 & 98.0 & 98.0 & 98.0 \\
\hline 2.4-D & 98.3 & 97.8 & 97.8 & 97.8 & 97.0 & 97.0 & 97.5 & 97.5 & 97.3 \\
\hline MCPP+dicamba & 97.8 & 98.0 & 97.8 & 97.5 & 98.0 & 97.8 & 98.3 & 97.8 & 97.3 \\
\hline Triasulfuron+dicamba & 97.5 & 98.0 & 97.5 & 97.8 & 97.5 & 97.0 & 97.3 & 97.3 & 97.8 \\
\hline 2.4-D+florasulam & 97.8 & 97.5 & 97.8 & 97.8 & 97.3 & 96.8 & 97.3 & 97.0 & 97.3 \\
\hline Amidosulfuron+iodosulfuronn & 97.5 & 97.8 & 98.0 & 98.8 & 97.0 & 97.5 & 97.5 & 97.8 & 97.5 \\
\hline Florasulam+flumetsulam & 97.5 & 97.5 & 97.8 & 97.5 & 98.0 & 97.5 & 97.8 & 97.0 & 97.8 \\
\hline LSD 0.05 & 1.6 & 1.1 & 1.1 & 1.4 & 1.4 & 1.6 & 1.5 & 1.5 & 1.3 \\
\hline LSD 0.01 & 2.1 & 1.5 & 1.5 & 1.9 & 1.9 & 2.1 & 2.0 & 2.0 & 1.8 \\
\hline
\end{tabular}

In $3^{\text {rd }}$ year (table 4) the highest barley seed germination $(97,5 \%)$ was measured at NS 293 variety treated with 2,4-D at first node stage, also, at Reks variety treated with florasulam+flumetsulam at second node stage. Lowest barley seed germination (95,8\%) was mesaured at NS 293 variety treated with florasulam+flumetsulam at second node stage. Also in this year the investigated herbicides did not significantly influence the barley seed germination.

Table 4. Influence of herbicides on barley seeds germination ( $3^{\text {rd }}$ year.)

\begin{tabular}{|c|c|c|c|c|c|c|c|c|c|}
\hline \multirow{4}{*}{ Variants } & \multicolumn{9}{|c|}{ Varieties } \\
\hline & \multicolumn{3}{|c|}{ Reks } & \multicolumn{3}{|c|}{ NS 293} & \multicolumn{3}{|c|}{ Egej } \\
\hline & 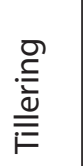 & $\begin{array}{l}\frac{0}{0} \\
\frac{0}{c} \\
\stackrel{\hbar}{=} \\
i=\end{array}$ & 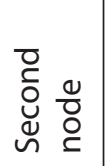 & 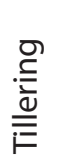 & 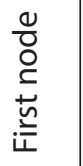 & 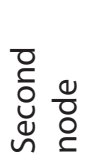 & 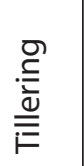 & 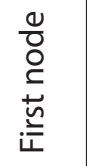 & 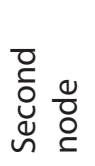 \\
\hline & $\%$ & $\%$ & $\%$ & $\%$ & $\%$ & $\%$ & $\%$ & $\%$ & $\%$ \\
\hline Weed free control & 97.0 & 97.0 & 97.0 & 96.8 & 96.8 & 96.8 & 97.5 & 97.5 & 97.5 \\
\hline 2,4-D & 96.3 & 96.5 & 96.3 & 96.8 & 97.5 & 96.5 & 96.8 & 96.5 & 96.5 \\
\hline MCPP+dicamba & 97.0 & 96.3 & 96.5 & 97.0 & 96.8 & 97.0 & 96.5 & 97.0 & 97.0 \\
\hline Triasufurone +dicamba & 96.8 & 96.8 & 97.0 & 96.5 & 97.0 & 96.8 & 97.0 & 96.5 & 97.0 \\
\hline 2,4-D+florasulam & 96.8 & 96.8 & 96.8 & 96.8 & 97.0 & 96.8 & 97.3 & 97.0 & 97.3 \\
\hline Amidosulfuron+iodosulfuronn & 96.5 & 96.8 & 97.3 & 97.0 & 97.3 & 97.0 & 97.0 & 97.0 & 97.3 \\
\hline Florasulam+flumetsulam & 96.5 & 97.0 & 97.5 & 97.0 & 97.3 & 95.8 & 97.3 & 96.8 & 96.8 \\
\hline LSD 0.05 & 1.4 & 1.2 & 1.5 & 1.4 & 1.1 & 1.5 & 1.3 & 1.2 & 1.2 \\
\hline LSD 0.01 & 1.8 & 1.7 & 2.1 & 1.9 & 1.6 & 2.0 & 1.8 & 1.6 & 1.6 \\
\hline
\end{tabular}


There is no differences between growth stages during the herbicide applications in all three years of testing (Table 5), so growth stages have no impact on barley seed germination. Similar results were reported by
(Danica et al.,1987) where examined herbicides do not have negative impact on wheat seed germination treated in tillering and shooting stage.

Table 5. Influence of growth stages on barley seeds germination.

\begin{tabular}{|c|c|c|c|c|c|c|c|c|c|}
\hline & \multicolumn{9}{|c|}{ Average of all herbicides } \\
\hline & \multicolumn{3}{|c|}{$1^{\text {st }}$ year } & \multicolumn{3}{|c|}{$2^{\text {nd }}$ year } & \multicolumn{3}{|c|}{$3^{\text {rd }}$ year } \\
\hline & $\frac{\widetilde{v}}{\widetilde{\varpi}}$ & 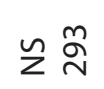 & 㞼 & $\begin{array}{l}\widetilde{\widetilde{\Xi}} \\
\widetilde{\otimes}\end{array}$ & $\Sigma \stackrel{n}{\sim}$ & 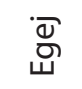 & $\frac{\widetilde{v}}{\widetilde{\widetilde{v}}}$ & $\Sigma \stackrel{n}{\sim}$ & অ্ণ্ড \\
\hline & $\%$ & $\%$ & $\%$ & $\%$ & $\%$ & $\%$ & $\%$ & $\%$ & $\%$ \\
\hline Tillering & 96.6 & 96.8 & 96.7 & 97.7 & 97.8 & 97.6 & 96.6 & 96.8 & 97.0 \\
\hline First node & 96.4 & 96.9 & 96.8 & 97.8 & 97.5 & 97.4 & 96.7 & 97.1 & 96.8 \\
\hline Second node & 96.6 & 96.8 & 96.8 & 97.8 & 97.3 & 97.5 & 96.9 & 96.6 & 97.0 \\
\hline
\end{tabular}

According Spasic (1972), the investigated herbicides including 2,4-D, MCPA, $M C P A+d i c a m b a$ and terbutrin have not negative impact on wheat seed germination.
Germination of galt barley variety was not affected by treating parent plants with MCPA, 2,4-D and metribuzine (Jeffery and John, 1984)

\section{CONCLUSIONS}

Based of the obtained results it can be concluded that the influence of herbicides on barley seed germination is not dependent on the growth stages during the application. If conditions do not allow herbicides to be used in the optimal period, it can be used no later than second node stage, without having a negative impact on the barley seed germination.

\section{REFERENCES}

Allen, F.C. (1966). Tolerance of barley to MCPA and dicamba. Proc. $19^{\text {th }}$ New Zeland Weed Pest Control Conference, 25-27.

Anonymous (2009). Agricultural statistics of Republic of Macedonia. Ministry for Agriculture, Forestry and Water Utilization, Goverment of Republic of Macedonia

Danica, D., Zdravko, K. \& Dusan, G. (1987). Effects of herbicides on wheat yield germination of wheat seeds. Fragmenta herbologica Jugoslavica, 16(1-2), 203$208(\mathrm{Sr})$.

Foley, E. M. (1985). Response differences of wheat (Triticum aestivum) and barley (Hordeum vulgare) to chlorsulfurone. Weed science, 34(1),17-21.

Friesen,H.A., Baenziger, H \& Keys, C. H. (1964). Morphological and cytological effects of dicamba on wheat and barley. Canadian Journal of Plant Science, 44, 288-294.
Friesen, H. A., Vanden, B., Keys, C. H., Dryden, R. D., Molberg, E. S. \& Siemens, B. (1968). Effect of time of application and dosage of dicamba on the tolerance of wheat, oats and barley. Canadian Journal of Plant Science, 48, 213-215.

Jeffery, S. C. \& John, A. D. (1984). Post emergence broad leaf in barley. Agricultural experiment station, School of Agriculture and Land resources Management, University of Alaska, Buletin 63, 1-16.

Randy, L. A. (1986). Metribuzine and chlorsulfurone effect on grain of treated winter wheat (Triticum aestivum). Weed science, 34, 734-737.

Rinella, J. M., Kells, J. J. Ward, W., R. (2001). Response of "Wakefield" winter wheat (Triticum aestivum) to Dicamba. Weed Technology, 15(3), 523-521.

Markovik, M. (1978). Effect of herbicides on winter wheat yields depending on 
the application phase. Yugoslavian Counseling of Weed Management with Airplanes and Helicopters, Osijek, 150$160(\mathrm{Sr})$.

Spasik, M. (1972). Investigation of the phytotoxic effects of herbicides on wheat. $X$
Yugoslavian Counseling for Weed Control, Novi Sad, 207-214 (Sr).

Tottman, D. R. (1976). Spray timing and the identification of cereal growth stages. Proceedings 1976 British Crop Conference-Weeds, 791-800.

\title{
ВЛИЈАНИЕ НА ХЕРБИЦИДИТЕ НА 'РТЛИВОСТА НА ЈАЧМЕНОТ ВО ЗАВИСНОСТ ОД ФАЗАТА НА ПОРАСТ
}

\author{
Гордана Глаткова ${ }^{1 *}$, Ментор Зеќири ${ }^{2}$ \\ 'Уким, Земјоделски Институт-Скопје, Република Северна Македонија \\ ${ }^{2}$ МЗШВ, Република Северна Македонија \\ *Контакт автор: gordana glatkova@yahoo.com
}

\section{Резиме}

Во текот на три години беа изведени испитувања кај три сорти јачмен (рекс, HC 293 и егеј) со 6 хербицидни варијанти (2,4-Д, МЦПП+дикамба, триасулфурон+дикамба, 2,4-Д+флорасулам, амидосулфурон+јодсулфурон и флорасулам+флуметсулам) во три различни фази на пораст (братење, прво коленце и второ коленце).

Целта на овој експеримент беше да се утврди влијанието на хербицидите аплицирани во три различни фази на пораст врз 'ртливоста на јачменот.

Во трите години на испитување хербицидите не влијаеа врз 'ртливоста на јачменот. Исто така, фазите на пораст на јачменот за време на третирањето немаа влијание врз 'ртливоста на семето јачмен. Ако условите не дозволуваат хербицидите да се употребат во оптималниот период, тие може да се аплицираат до фаза појава на второ коленце без да имаат негативно влијание врз 'ртливоста на јачменот.

Клучни зборови: јачмен, сорти, хербичиди, фази на пораст, 'ртливост 